\title{
Channel Quality Prediction Based on Bayesian Inference in Cognitive Radio Networks
}

\author{
Xiaoshuang Xing ${ }^{1}$, Tao Jing ${ }^{1}$, Yan Huo ${ }^{1}$, Hongjuan $\mathrm{Li}^{2}$, Xiuzhen Cheng ${ }^{2}$ \\ ${ }^{1}$ School of Electronics and Information Engineering, Beijing Jiaotong University, Beijing, China \\ ${ }^{2}$ Department of Computer Science, The George Washington University, Washington DC, USA \\ E-mail: \{10120170,tjing,yhuo\}@bjtu.edu.cn, \{hongjuan,cheng\}@gwu.edu
}

\begin{abstract}
The problem of channel quality prediction in cognitive radio networks is investigated in this paper. First, the spectrum sensing process is modeled as a Non-Stationary Hidden Markov Model (NSHMM), which captures the fact that the channel state transition probability is a function of the time interval the primary user has stayed in the current state. Then the model parameters, which carry the information about the expected duration of the channel states and the spectrum sensing accuracy (detection accuracy and false alarm probability) of the $\mathrm{SU}$, are estimated via Bayesian inference with Gibbs sampling. Finally, the estimated NSHMM parameters are employed to design a channel quality metric according to the predicted channel idle duration and spectrum sensing accuracy. Extensive simulation study has been performed to investigate the effectiveness of our design. The results indicate that channel ranking based on the proposed channel quality prediction mechanism captures the idle state duration of the channel and the spectrum sensing accuracy of the SUs, and provides more high quality transmission opportunities and higher successful transmission rates at shorter spectrum waiting times for dynamic spectrum access.

Index Terms-Channel quality prediction; cognitive radio networks; non-stationary HMM; Bayesian inference.
\end{abstract}

\section{INTRODUCTION}

Cognitive radio [1]-[9] has been envisioned as a promising approach to alleviate the spectrum scarcity problem as well as to increase the efficiency of spectrum utilization. In cognitive radio networks (CRNs), secondary users (SU) are allowed to opportunistically access the primary channels on a noninterference basis. However, the quality of the channels may differ significantly and overlooking high-quality ones may drastically decrease the spectrum efficiency. Thus in this paper we tackle the challenges of channel quality prediction to enhance the efficiency of dynamic spectrum access.

Our objective is to estimate the parameters of the channels from the spectrum sensing results of the secondary users. We intend to identify for each SU the channel that has high detection accuracy, low false alarm probability, and long idle duration. For this purpose, we model the process of spectrum sensing as a Non-Stationary Hidden Markov Model (NSHMM). The NSHMM is a more accurate model compared to the stationary HMM as in reality, the probabilities of state transitions are a function of the time interval the primary user has stayed in the current state. In this paper the model parameters are estimated via Bayesian inference with Gibbs sampling, which provides a viable approach to infer the expected duration of the channel states and the spectrum sensing accuracy of the SUs. We also propose a metric, which utilizes the estimated NSHMM parameters, to evaluate the channel quality according to the predicted channel idle duration and the spectrum sensing accuracy quantified by the estimated spectrum detection probability and false alarm rate. The expected idle duration is determined by the PU activities while the spectrum sensing accuracy is mainly affected by the spectrum sensing technology and the communication environment.

The main contributions of the paper are summarized as follows. First, we employ the Non-Stationary HMM to model the spectrum sensing process and infer the model parameters through Bayesian inference with Gibbs sampling. To our best knowledge, existing works [10], [11] all adopt the stationary HMM for spectrum detection and prediction, which can not capture the busy/idle duration of the primary channels. We are the first to employ the NSHMM to model the channel status in a CRN. Second, a novel channel quality evaluation metric is proposed to help the SU select a channel with high quality according to the predicted channel idle duration and spectrum sensing accuracy. Our metric also considers the preference of the secondary users, i.e., a SU has the freedom to select the channel with the longest estimated idle duration or the one it possesses the highest spectrum sensing accuracy (high detection probability and low false alarm rate). Third, we perform an extensive simulation study to validate our NSHMM model and investigate the impact of various model parameters on the expected channel quality. We also consider two example application scenarios, with the first one addressing the channel ranking based on the predicted channel quality and the second one tackling the efficiency of dynamic spectrum access according to the channel ranking.

The rest of the paper is organized as follows. The related work is reviewed in Section II. The network model and necessary preliminaries are presented in Section III. The prior probability distributions of the NSHMM parameters are derived in Section IV. Channel quality prediction based on Bayesian inference is proposed in Section V. The simulation results are reported in Section VI, and the conclusion of the paper is given in Section VII.

\section{RELATED WORK}

Channel quality prediction plays a critical role in enhancing the efficiency of wireless resource utilization. A rich body of research on channel quality estimation/prediction has been 
performed in OFDM systems [12], mobile cellular networks [13], adaptive code modulation [14], and so on. But the existing technical approaches can not be applied to CRNs as SUs can only occupy a channel when it is not utilized by primary users, which implies that channel availability has to be considered when estimating the channel quality in a CRN. More specifically, we have to take into account the idle duration of the channel, i.e., the length of the time the channel can be used by a SU, when evaluating the channel quality.

Strictly speaking, channel quality prediction for CRNs has never been addressed to our best knowledge. Recent research [15]-[18] propose to take advantage of channel quality information to enhance the efficiency of dynamic spectrum access in CRNs but none of them considers the important idle durations of the channels. In [15] and [16], the utility of secondary users is modeled as a function of the channel quality, but how to estimate and evaluate the channel quality is not discussed. Channel-aware distributed scheduling is considered in [17], [18], where the channel quality is mainly determined by the transmission rate. Note that the works mentioned above all require messages to be passed between secondary users, which increases the energy consumption and transmission overhead. The channel quality prediction scheme proposed in this paper, on the other hand, employs only the local spectrum sensing results to infer the parameters of the channels by each SU; thus completely avoiding the overhead of message exchange. Moreover, our approach can predict the estimated channel duration, which can facilitate the SU to select the channel that could stay idle for a long time.

\section{Network Model AND Preliminaries}

\section{A. Network Model and Problem Description}

In this paper, we consider a time-slotted cognitive radio network. The busy/idle status of a channel is detected by a SU via energy detection based spectrum sensing techniques [19], [20] at the beginning of each time slot. If the channel is idle, the SU starts its transmission (facilitated by multiple access control) until the end of the slot; otherwise, the SU recesses and starts to perform spectrum sensing again at the beginning of the next time slot. We assume that the channel status is stable within a time slot; that is, a channel state lasts at least one slot. This process is illustrated in Fig. 1, in which the SU obtains an observation $o_{t}$ on the true state $q_{t}$ of the channel at each time slot $t$. The observation $o_{t}$ is the local decision of the SU regarding the primary channel status. An SU is said to make a detection if $o_{t}=b u s y$ and $q_{t}=b u s y$, and said to make a false alarm if $o_{t}=$ busy and $q_{t}=i d l e$. The detection probability $P_{d}$ and false alarm probability $P_{f}$ are given respectively by (1) and (2) according to [20], [21].

$$
\begin{aligned}
P_{d}= & e^{-\frac{\chi}{2}} \sum_{n=0}^{m-2} \frac{1}{n !}\left(\frac{\chi}{2}\right)^{n}+\left(\frac{1+\bar{\xi}}{\bar{\xi}}\right)^{m-1} \\
& {\left[e^{-\frac{\chi}{2(1+\bar{\xi})}}-e^{-\frac{\chi}{2}} \sum_{n=0}^{m-2} \frac{1}{n !}\left(\frac{\chi \bar{\xi}}{2(1+\bar{\xi})}\right)^{n}\right] }
\end{aligned}
$$

$$
P_{f}=\frac{\Gamma\left(m, \frac{\chi}{2}\right)}{\Gamma(\mu)}
$$

where $\chi$ is the threshold of the energy detector, $m$ is the time bandwidth product, $\bar{\xi}$ is the average SNR of the SU on the primary channel, $\Gamma(.,$.$) is the incomplete gamma function,$ and $\Gamma($.$) is the gamma function. Note that \bar{\xi}$ is defined as $\bar{\xi}=\frac{P_{P U} h_{P S}}{\varsigma^{2}}$, with $P_{P U}$ being the transmit power of the PU, $\varsigma^{2}$ being the Gaussian noise variance, and $h_{P S}=\frac{\kappa}{d_{P S}^{\mu}}$ being the path loss between the PU and the SU; here $\kappa$ is the path loss constant, $\mu$ is the path loss exponent, and $d_{P S}$ is the distance between the PU and the SU.

The detection probability and false alarm probability indicate the spectrum sensing accuracy of the SU; and the

\begin{tabular}{|c|c|c|c|c|c|c|}
\hline slots & $t=1$ & $t=2$ & $\cdots \cdots$ & $t=n$ & $\cdots \cdots$ & $t=T$ \\
\hline True states & $q_{1}$ & $q_{2}$ & $\cdots \cdots$ & $q_{n}$ & $\cdots \cdots$ & $q_{T}$ \\
\hline observations & $o_{I}$ & $\mathrm{O}_{2}$ & $\ldots \ldots$ & $o_{n}$ & $\cdots \cdots$ & $o_{T}$ \\
\hline
\end{tabular}
spectrum sensing accuracy has significant influence on the spectrum utilization efficiency of the SU over the channel.

Fig. 1. An illustration of the traditional spectrum sensing process.

The spectrum sensing process illustrated in Fig. 1 can be modeled as a Hidden Markov Model (HMM). In [22], the existence of a Markov chain in the channel utilization of a PU over the time domain has been validated. Therefore, the channel occupancy states $Q=\left\{q_{1}, q_{2}, \cdots, q_{T}\right\}$, determined by the PU activities (on or off), form a Markov process. These states are hidden since they are not directly observable. On the other hand, SU generates its observation sequence $O=$ $\left\{o_{1}, o_{2}, \cdots, o_{T}\right\}$ based on its spectrum sensing results, which form the set of observation states. This Markov process is a normal random process that depends on the activities of the PU and the spectrum sensing accuracy of the SU. Thus the spectrum sensing procedure can be modeled as a HMM. Moreover, the hidden state space is defined as $S=\left\{s_{0}, s_{1}\right\}$, with $s_{0}=0$ and $s_{1}=1$ indicating that the channel is idle and busy, respectively. Similarly, the observation state space is defined as $V=\left\{v_{0}, v_{1}\right\}$, with $v_{0}=0$ and $v_{1}=1$ indicating that the spectrum sensing result is idle and busy, respectively.

In this paper, we model the spectrum sensing process as a nonstationary HMM (NSHMM) and infer its parameters through Bayesian inference with Gibbs sampling. Then the inferred parameters are employed to predict the channel quality, which takes into account both the idle durations of the channels and the spectrum sensing accuracy of the SUs over the channels. For better understanding, we outline the preliminary knowledge about HMM and Bayesian inference in the following subsections.

\section{B. Conventional HMM and Non-Stationary HMM}

Consider a scenario with the hidden space $S=\left\{s_{0}=0, s_{1}=1\right\}$ and the observation space $V=\left\{v_{0}=0, v_{1}=1\right\}$. A conventional 


\begin{tabular}{|c|c|c|c|c|}
\multicolumn{1}{c}{$\mathbf{k}_{1}$} & \multicolumn{1}{c}{$\mathbf{l}_{1}$} & $\mathbf{k}_{2}$ & \multicolumn{1}{l}{$\mathbf{l}_{2}$} \\
\hline 0(idle) & 1(busy) & 0(idle) & 1(busy) & $\ldots \ldots$. \\
\hline
\end{tabular}

Fig. 2. An example of a state sequence $Q$, where $k_{i}, i=1,2 \ldots$ denotes a segment with $k_{i}$ adjacent 0 's (idle state) and $l_{i}, i=1,2 \ldots$ denotes a segment with $l_{i}$ adjacent 1 's (busy state).

HMM can be described by its parameters $\Lambda_{C}=\left(\Pi_{C}, A_{C}, B_{C}\right)$, where $\Pi_{C}$ is the initial state probability distribution: $\Pi_{C}=\left[\pi_{i}\right]_{1 \times 2}, \pi_{i}=P\left(q_{1}=s_{i}\right), i \in\{0,1\} ; A_{C}$ is the state transition probability matrix: $A_{C}=\left[a_{i j}\right]_{2 \times 2}, a_{i j}=P\left(q_{t+1}=\right.$ $\left.s_{j} \mid q_{t}=s_{i}\right), i, j \in\{0,1\}$; and $B_{C}$ is the emission probability matrix: $B_{C}=\left[b_{j k}\right]_{2 \times 2}, b_{j k}=P\left(o_{t}=v_{k} \mid q_{t}=s_{j}\right), j, k \in\{0,1\}$. According to (1) and (2), it is obvious that $b_{01}=P_{f}$ and $b_{11}=P_{d}$.

The parameters of a conventional HMM are all timestationary. For example, the probability that the state sequence shown in Fig. 2 holds can be expressed as:

$$
P\left(Q \mid \Lambda_{C}\right)=\pi(0) a_{00}^{k_{1}-1} a_{01} a_{11}^{l_{1}-1} a_{10} a_{00}^{k_{2}-1} a_{01} a_{11}^{l_{2}-1} \ldots
$$

It can be seen that the transition probability $a_{i j}$ is a static value independent of the duration of the state $s_{i}$. No matter how many slots the state $s_{i}$ has been lasted, the probability that the next state is $s_{i}\left(s_{j}\right)$ is always $a_{i i}\left(a_{i j}\right)$. Intuitively, this is not reasonable as in practice it is more often the case that the transition probability is a function of $\tau$, the duration of a particular state. Define $a_{i j}(\tau)$ as the probability that the system switches from $s_{i}$ to $s_{j}$, given that the system has already been on state $s_{i}$ for $\tau$ consecutive time slots [23]:

$$
a_{i j}(\tau)=P\left(q_{t}=s_{j} \mid q_{t-1}=q_{t-2}=\cdots=q_{t-\tau}=s_{i}\right)
$$

Therefore, the transition probabilities are functions of time; thus they are non-static. The HMMs with non-static transition probabilities are referred as non-stationary hidden Markov models (NSHMMs). A NSHMM can also be described by its parameters $\Lambda_{N}=\left(\Pi_{N}, A_{N}(\tau), B_{N}\right)$. Here, $\Pi_{N}=\left[\pi_{i}\right]_{1 \times 2}$ and $B_{N}=\left[b_{j k}\right]_{2 \times 2}$ have the same expression as those of the conventional HMM while $A_{N}(\tau)=\left[a_{i j}(\tau)\right]_{2 \times 2}$.

It has been proved in [24], [25] that the duration of a channel state (idle or busy) is exponentially distributed. Let $d_{i}$ denote the duration of the state $s_{i}, i=0,1$. Then the probability density function of $d_{i}$ is:

$$
f\left(d_{i}\right)=\lambda_{i} e^{-\lambda_{i} d_{i}}
$$

where $\lambda_{i}$ is the rate parameter of the exponential distribution with $E\left[d_{i}\right]=1 / \lambda_{i}$.

Denote by $t_{s}$ the length of a time slot. Then the self transition probabilities $a_{i i}(\tau), i=0,1$, can be given by:

$$
\begin{aligned}
a_{i i}(\tau) & =\mathrm{P}\left(\text { duration of state } s_{i}>\tau\right) \\
& =1-\int_{d_{i}=0}^{\tau t_{s}} f\left(d_{i}\right) d d_{i} \\
& =e^{-\lambda_{i} \tau t_{s}}
\end{aligned}
$$

Since there are only two states in our hidden space, the outward state transition probabilities $a_{i j}(\tau), j=0,1$ and $j \neq$ $i$, can be given as

$$
\begin{aligned}
a_{i j}(\tau) & =1-a_{i i}(\tau) \\
& =1-e^{-\lambda_{i} \tau t_{s}}
\end{aligned}
$$

\section{Preliminary Knowledge About Bayesian Inference}

In this subsection, we briefly describe the Bayesian inference procedure to derive the probability distributions of the system parameters. To proceed, it is necessary to introduce three basic concepts, namely prior probability distribution, likelihood function, and posterior probability distribution.

Definition 1. In Bayesian inference, a prior probability distribution (also known as prior) of a system parameter $\theta$, denoted by $P(\theta)$, is often an experimental subjective assessment about $\theta$ before the data is taken into account.

Definition 2. Given some observed data $X=$ $\left\{x_{1}, x_{2}, \cdots, x_{n}\right\}$, the Likelihood function of parameter $\theta$, denoted by $L(\theta \mid X)$, is defined to be the probability of the observed data given that parameter. That is, $L(\theta \mid X)=P(X \mid \theta)$. A likelihood function is often used to estimate the system parameter from a set of statistical data.

Definition 3. In Bayesian inference, the posterior probability distribution (also known as posterior) is the distribution of a system parameter $\theta$ conditioned on the data $X=$ $\left\{x_{1}, x_{2}, \cdots, x_{n}\right\}$ observed from an experiment or survey. Therefore, the posterior probability distribution of $\theta$ can be denoted by $P(\theta \mid X)$.

Given Definitions 1-3, we get the fowling expression according to Bayes' rule:

$$
\begin{aligned}
P(\theta \mid X) & =\frac{P(X \mid \theta) \cdot P(\theta)}{P(X)} \\
& \propto P(X \mid \theta) \cdot P(\theta)
\end{aligned}
$$

where $\propto$ means "proportional to".

Bayesian inference intends to compute the posterior according to (8), which indicates that the posterior can be derived from the prior probability distribution and the likelihood function. Hence, the primary problem is to specify a prior for each parameter. The selection of a prior has a significant influence on the complexity of posterior computation. Therefore, a special kind of prior called conjugate prior is adopted in this paper to reduce the posterior calculation complexity:

Definition 4. The prior probability distribution and the posterior probability distribution are conjugate if the posterior $P(\theta \mid X)$ is in the same family as the prior $P(\theta)$. In this case, the prior is called a conjugate prior for the likelihood.

Choosing a reasonable conjugate prior is often an effective way to simplify the posterior calculation. Thus in this paper, we first derive a reasonable conjugate prior for each system parameter through theoretical analysis. 


\section{PRior Derivation For NSHMM Parameters}

According to the description in Section III-C, prior probability distributions should be specified before Bayesian inference can be conducted. In this section, we intend to define a reasonable conjugate prior for each parameter of the NSHMM through theoretical analysis.

\section{A. Conjugate Prior Derivation for $\Pi_{N}$}

Assume that the initial channel state is a random binary variable $X$ that takes value 1 with probability $\pi_{1}$ and value 0 with probability $\pi_{0}=1-\pi_{1}$. It is obvious that $X$ follows a Bernoulli distribution, denoted by $X \sim \operatorname{Bernoulli}\left(\pi_{1}\right)$. Given an observed data $X=x$, the likelihood function of $\pi_{1}$ is

$$
P\left(X=x \mid \pi_{1}\right)=\pi_{1}^{x}\left(1-\pi_{1}\right)^{1-x}
$$

According to [26], we can adopt a uniform distribution between 0 and 1 as the prior for $\pi_{1}$ if we have no knowledge of its probability distribution. That is, $\pi_{1} \sim U(0,1)$ and

$$
P\left(\pi_{1}\right)= \begin{cases}1, & \text { if } 0 \leq \pi_{1} \leq 1 \\ 0, & \text { otherwise }\end{cases}
$$

Then the denominator in (8) can be calculated by

$$
\begin{aligned}
P(X=x) & =\int_{\pi_{1}} P\left(X=x \mid \pi_{1}\right) P\left(\pi_{1}\right) d \pi_{1} \\
& =\int_{\pi_{1}=0}^{1} \pi_{1}^{x}\left(1-\pi_{1}\right)^{1-x} d \pi_{1} \\
& =\frac{\Gamma(x+1) \Gamma(2-x)}{\Gamma(3)}
\end{aligned}
$$

Therefore, the posterior probability distribution of $\pi_{1}$ is

$$
\begin{aligned}
P\left(\pi_{1} \mid X=x\right) & =\frac{P\left(X=x \mid \pi_{1}\right) \cdot P\left(\pi_{1}\right)}{P(X=x)} \\
& =\frac{\Gamma(3)}{\Gamma(x+1) \Gamma(2-x)} \pi_{1}^{x}\left(1-\pi_{1}\right)^{1-x} \\
& =\frac{\Gamma(x+1+2-x)}{\Gamma(x+1) \Gamma(2-x)} \pi_{1}^{x+1-1}\left(1-\pi_{1}\right)^{2-x-1}
\end{aligned}
$$

It can be seen that the posterior of $\pi_{1}$ is a Beta distribution $\operatorname{Beta}(x+1,2-x)$. Note that a uniform distribution can also be considered as a special Beta distribution $\operatorname{Beta}(1,1)$; therefore a Beta distribution is a reasonable conjugate prior when the likelihood is a Bernoulli distribution.

\section{B. Conjugate Prior Derivation for $A_{N}(\tau)$}

According to our network model described in Section III, there is a hidden state sequence $Q=\left\{q_{1}, q_{2}, \cdots, q_{T}\right\}$ in the spectrum sensing process. Let $Y_{i}$ be a random variable denoting the average duration of the state $s_{i}$ in the sequence $Q$, and $y_{i}$ be a specific value of $Y_{i}$. According to [24], [25], [27], $Y_{i}$ follows an Exponential distribution, i.e., $Y_{i} \sim \operatorname{Exp}\left(\lambda_{i}\right)$. From (5), the likelihood function of $\lambda_{i}$ is:

$$
P\left(y_{i} \mid \lambda_{i}\right)=f\left(y_{i}\right)=\lambda_{i} e^{-\lambda_{i} y_{i}}
$$

The expected duration of the state $s_{i}, E\left[y_{i}\right]$, satisfies $1 \leq$ $E\left[y_{i}\right]=\frac{1}{\lambda_{i}}<\infty$, so that $0<\lambda_{i} \leq 1$.

In Bayesian inference, the Gamma distribution is the conjugate prior to many likelihood distributions such as Exponential [28]. Thus in the following we derive the posterior of $\lambda_{i}$ assuming that the Gamma distribution is a conjugate prior for $\lambda_{i}$, i.e., $\lambda_{i} \sim \operatorname{Gamma}(a, b)$. Then

$$
\begin{aligned}
P\left(\lambda_{i}\right) & =f\left(\lambda_{i}\right)=\frac{1}{b^{a}} \frac{1}{\Gamma(a)} \lambda_{i}^{a-1} e^{-\frac{\lambda_{i}}{b}} \\
P_{i}\left(\lambda_{i} \mid y_{i}\right) & \propto P\left(y_{i} \mid \lambda_{i}\right) P\left(\lambda_{i}\right) \\
& \propto \lambda_{i}^{a+1-1} e^{-\frac{\lambda_{i}}{b y_{i}+1}} \\
& \sim \operatorname{Gamma}\left(a+1, \frac{b}{b y_{i}+1}\right)
\end{aligned}
$$

We conclude that the Gamma distribution is indeed a reasonable conjugate prior for $\lambda_{i}$, which could be used to derive the probability distributions of $a_{i i}(\tau)$ and $a_{i j}(\tau)$ for $i, j \in\{0,1\}$.

\section{Conjugate Prior Derivation for $B_{N}$}

Consider the hidden state sequence $Q=\left\{q_{1}, q_{2}, \cdots, q_{T}\right\}$, $q_{t} \in S, t \in\{1, \cdots, T\}$, and the corresponding observation sequence $O=\left\{o_{1}, o_{2}, \cdots, o_{T}\right\}, o_{t} \in V, t \in\{1, \cdots, T\}$. Let $n_{i}, i=0,1$, be the number of the state $s_{i}$ in $Q$. Define a random variable $Z_{i i}$ to denote the number of observations $v_{i}$ in state $s_{i}$. It is obvious that $Z_{i i}$ follows a Binomial distribution: $Z_{i i} \sim \operatorname{Binomial}\left(n_{i}, b_{i i}\right)$. Then the likelihood of $b_{i i}$ is

$$
\begin{aligned}
P\left(Z_{i i}=z \mid b_{i i}\right) & =C_{n_{i}}^{z} b_{i i}^{y}\left(1-b_{i i}\right)^{n_{i}-z} \\
& \propto b_{i i}^{z}\left(1-b_{i i}\right)^{n_{i}-z}, z=0,1, \cdots, n
\end{aligned}
$$

Assuming that the prior for $b_{i i}$ is uniformly distributed in [0,1], i.e., $P\left(b_{i i}\right)=1,0 \leq b_{i i} \leq 1$, according to [26]. Then the posterior of $b_{i i}$ can be given as follows:

$$
\begin{aligned}
P_{i}\left(b_{i i} \mid Z_{i i}=z\right) & \propto P\left(Z_{i i}=z \mid b_{i i}\right) P\left(b_{i i}\right) \\
& =b_{i i}^{z}\left(1-b_{i i}\right)^{n_{i}-z} \\
& =b_{i i}^{(z+1)-1}\left(1-b_{i i}\right)^{\left(n_{i}-z+1\right)-1} \\
& \sim \operatorname{Beta}\left(z+1, n_{i}-z+1\right)
\end{aligned}
$$

Therefore, we conclude that Beta distribution is a reasonable conjugate prior for $b_{i i}$.

\section{Prior Specification for the NSHMM Parameters}

In the previous subsections, we identify an appropriate conjugate prior for each NSHMM parameter. In this subsection, we give specific definitions for these prior probability distributions.

1) Define $\pi_{1} \sim \operatorname{Beta}(\alpha, \beta)$ and $\pi_{0}=1-\pi_{1}$.

2) Define $\lambda_{i} \sim \operatorname{Gamma}\left(\eta_{i}, \gamma_{i}\right), i=0,1$, and

$$
\begin{aligned}
& a_{i i}(\tau)=e^{-\lambda_{i} \tau t_{s}} \\
& a_{i j}(\tau)=1-e^{-\lambda_{i} \tau t_{s}} \quad j=0,1, i \neq j
\end{aligned}
$$

3) Define $b_{i i} \sim \operatorname{Beta}\left(\varepsilon_{i}, \sigma_{i}\right), i=0,1$ and $b_{i j}=1-b_{i i}, j=$ $0,1, i \neq j$. 
The prior parameters include $\alpha, \beta, \eta_{i}, \gamma_{i}, \varepsilon_{i}, \sigma_{i}, i=0,1$, which are called hyperparameters. The hyperparameters can be selected using the experimental knowledge about the problem [26]. In this paper, the experiential average value of the parameters are used for hyperparameter selection. For example, we know from experience that the average value of $\pi_{1}$ is $\bar{\pi}_{1}$. Since $\pi_{1} \sim \operatorname{Beta}(\alpha, \beta)$, the expectation of $\pi_{1}$, denoted by $E\left[\pi_{1}\right]$, equals $\frac{\alpha}{\alpha+\beta}$. Thus we can obtain the following equation:

$$
\frac{\alpha}{\alpha+\beta}=\bar{\pi}_{1}
$$

It is clear that we can draw a liner relationship between $\alpha$ and $\beta$. To obtain the specific values for $\alpha$ and $\beta$, we should take the variance into consideration. The variance of $\pi_{1}$, denoted by $\operatorname{Var}\left[\pi_{1}\right]$ can be expressed as:

$$
\operatorname{Var}\left[\pi_{1}\right]=\frac{\alpha \beta}{(\alpha+\beta)^{2}(\alpha+\beta+1)}
$$

If we are confident about the experiential average value $\bar{\pi}_{1}$, we can choose appropriate $\alpha$ and $\beta$ values that lead to a small variance $\operatorname{Var}\left[\pi_{1}\right]$; otherwise, a big variance might be preferred. However, since Bayesian inference is one of the machine learning approaches, the confidence on the experiential values increases with the learning time, i.e., the time that the SU has been spending on observing the channel occupancy status. Therefore, $\operatorname{Var}\left[\pi_{1}\right]$ can be determined according to the learning time of the SU. Then, $\alpha$ and $\beta$ can be drawn from (21) and (22).

A similar procedure can be applied to determine the hyperparameters $\eta_{i}, \gamma_{i}, \varepsilon_{i}$ and $\sigma_{i}$, for $i=0,1$.

\section{Channel Quality Estimation Based on Bayesian INFERENCE}

\section{A. Bayesian Inference with Gibbs Sampling}

After the determination of the priors, Bayesian inference can be conducted to derive the posteriors of the NSHMM parameters. Since Gibbs sampling is a candidate approach to reduce the computation complexity of Bayesian inference [23], we intend to implement Bayesian inference via a Gibbs sampling procedure in this section.

Initially, we set the hyperparameters of the priors according to the procedure given in Section IV-D. Specifically, we assume that $\pi_{1} \sim \operatorname{Beta}\left(\alpha^{0}, \beta^{0}\right), a_{i i} \sim \operatorname{Bet} a\left(\eta_{i}^{0}, \gamma_{i}^{0}\right)$, and $b_{i i} \sim \operatorname{Beta}\left(\varepsilon_{i}^{0}, \sigma_{i}^{0}\right)$, where $i=0,1 . Q^{0}$ is generated from the specified hyperparameters. We will perform $K$ iterations to derive the posteriors. The hyperparameters for the $k$-th iteration are denoted by $\alpha^{k}, \beta^{k}, \eta_{i}^{k}, \gamma_{i}^{k}, \varepsilon_{i}^{k}, \sigma_{i}^{k}$, for $i=0,1$.

According to Gibbs sampling, at iteration $k$, the probability distributions derived from iteration $k-1$ can be considered as prior probability distributions, and the parameters obtained from iteration $k-1$ can be considered as observations. Then the following steps are carried out to derive the posterior probability distributions for the $k$ th iteration:
Step 1:

$$
\begin{aligned}
\pi_{1}^{k} \sim & P\left(\pi_{1} \mid A_{N}^{k-1}, B_{N}^{k-1}, Q^{k-1}, O\right) \\
= & P\left(\pi_{1} \mid Q^{k-1}\right) \\
\propto & P\left(q_{1}^{k-1} \mid \pi_{1}\right) P\left(\pi_{1}\right) \\
= & \left(\pi_{1}\right)_{1}^{q_{1}^{k-1}}\left(1-\pi_{1}\right)^{\left(1-q_{1}^{k-1}\right)} \\
& \cdot \frac{\Gamma\left(\alpha^{k-1}+\beta^{k-1}\right)}{\Gamma\left(\alpha^{k-1}\right) \Gamma\left(\beta^{k-1}\right)}\left(\pi_{1}\right)^{\alpha^{k-1}-1}\left(1-\pi_{1}\right)^{\beta^{k-1}-1} \\
\propto & \left(\pi_{1}\right)^{\alpha^{k-1}+q_{1}^{k-1}-1}\left(1-\pi_{1}\right)^{\beta^{k-1}+1-q_{1}^{k-1}-1} \\
\sim & \operatorname{Beta}\left(\alpha^{k-1}+q_{1}^{k-1}, \beta^{k-1}+1-q_{1}^{k-1}\right)
\end{aligned}
$$

Randomly draw a specific value for $\pi_{1}^{k}$ from the derived posterior probability distribution $\operatorname{Beta}\left(\alpha^{k-1}+q_{1}^{k-1}, \beta^{k-1}+\right.$ $1-q_{1}^{k-1}$ ), and then we get:

$$
\begin{gathered}
\pi_{0}^{k}=1-\pi_{1}^{k} \\
\Pi_{N}^{k}=\left(\pi_{0}^{k}, \pi_{1}^{k}\right)
\end{gathered}
$$

Step 2:

$$
\begin{aligned}
\lambda_{i}^{k} & \sim P\left(\lambda \mid \Pi_{N}^{k}, B_{N}^{k-1}, Q^{k-1}, O\right) \\
& \propto \lambda_{i} e^{-\lambda_{i} \delta_{i}} \cdot \lambda^{\eta_{i}^{k-1}-1} e^{-\frac{\lambda_{i}}{\gamma_{i}^{k-1}}} \\
& =\lambda^{\eta_{i}^{k-1}+1-1} e^{-\frac{\lambda_{i}}{\frac{\gamma_{i}^{k-1}}{1+\gamma_{i}^{k-1} \delta_{i}}}} \\
& \sim \operatorname{Gamma}\left(\eta_{i}^{k-1}+1, \frac{\gamma_{i}^{k-1}}{1+\gamma_{i}^{k-1} \delta_{i}}\right)
\end{aligned}
$$

where $\delta_{i}$ is the average duration of the state $s_{i}$ in the state sequence $Q^{k-1}$.

Randomly draw $\lambda_{i}, i=0,1$, from the posterior probability distribution, and calculate the self transition probabilities $a_{i i}^{k}(\tau)$ for $i=0,1$, and the outward state transition probabilities $a_{i j}^{k}(\tau)$ for $j=0,1, j \neq i$, according to (6) and (7), respectively. Finally, we obtain

$$
A_{N}^{k}(\tau)=\left(\begin{array}{cc}
a_{00}^{k}(\tau) & a_{01}^{k}(\tau) \\
a_{10}^{k}(\tau) & a_{11}^{k}(\tau)
\end{array}\right)
$$

Step 3:

$$
\begin{aligned}
b_{i i}^{k} \sim & P\left(b_{i i}^{k} \mid \Pi_{N}^{k}, A_{N}^{k}, Q^{k-1}, O\right) \\
= & P\left(b_{i i}^{k} \mid Q^{k-1}, O\right) \\
\propto & C_{n_{i}}^{\omega_{i}}\left(b_{i i}^{k-1}\right)^{\omega_{i}}\left(1-b_{i i}\right)^{n_{i}-\omega_{i}} \\
& \cdot \frac{\Gamma\left(\varepsilon_{i}^{k-1}+\sigma_{i}^{k-1}\right)}{\Gamma\left(\varepsilon_{i}^{k-1}\right) \Gamma\left(\sigma_{i}^{k-1}\right)}\left(b_{i i}^{k-1}\right)^{\varepsilon_{i}^{k-1}-1}\left(1-b_{i i}^{k-1}\right)^{\sigma_{i}^{k-1}-1} \\
\propto & \left(b_{i i}^{k-1}\right)^{\varepsilon_{i}^{k-1}+\omega_{i}-1}\left(1-b_{i i}^{k-1}\right)^{\sigma_{i}^{k-1}+n_{i}-\omega_{i}-1} \\
\sim & \operatorname{Beta}\left(\varepsilon_{i}^{k-1}+\omega_{i}, \sigma_{i}^{k-1}+n_{i}-\omega_{i}\right)
\end{aligned}
$$

where $n_{i}$ is the number of the state $s_{i}$ in the state sequence $Q^{k-1}$, and $\omega_{i}$ is the number of symbols $v_{i}$ in the state $s_{i}$. 
Randomly draw $b_{i i}^{k}, i=0,1$, from the posterior probability distribution, and then

$$
B_{N}^{k}=\left(\begin{array}{cc}
b_{00}^{k} & b_{01}^{k} \\
b_{10}^{k} & b_{11}^{k}
\end{array}\right)=\left(\begin{array}{cc}
b_{00}^{k} & 1-b_{00}^{k} \\
1-b_{11}^{k} & b_{11}^{k}
\end{array}\right)
$$

Step 4:

$$
q_{1}^{k} \sim \operatorname{Bernoulli}\left(\pi_{1}^{k}\right)
$$

For $t \geq 2$,

$$
\begin{aligned}
q_{t}^{k} \sim & P\left(q_{t}^{k} \mid \Pi_{N}^{k}, A_{N}^{k}, B_{N}^{k}, O, q_{t-1}^{k}, q_{t+1}^{k}-1, \cdots, q_{T}^{k-1}\right) \\
= & a_{q_{t-1}^{k} q_{t}^{k}}\left(\tau\left(q_{t-1}^{k}\right)\right) \cdot a_{q_{t}^{k} q_{t+1}^{k-1}}\left(\tau\left(q_{t}^{k}\right)\right) \\
& \cdot \prod_{i=t+1}^{T-1} a_{q_{i}^{k-1} q_{i+1}^{k-1}}\left(\tau\left(q_{i}^{k-1}\right)\right) \cdot b_{q_{t}^{k} o_{t}}
\end{aligned}
$$

where $\tau(q)$ is the duration of the state $q$.

We randomly draw $q_{t}^{k}, t=1,2 \cdots, T$, from the posterior and get the state sequence of iteration $k$ :

$$
Q^{k}=\left(q_{1}^{k}, q_{2}^{k}, \cdots, q_{T}^{k}\right)
$$

After $K$ iterations, we get the estimated parameters of the NSHMM, denoted by $\widehat{\Lambda}_{N}=\left(\widehat{\Pi}_{N}, \widehat{A}_{N}(\tau), \widehat{B}_{N}\right)$, and the estimated rate parameter of the Exponential distribution of the state duration, denoted by $\widehat{\lambda}_{i}, i=0,1$. In the next subsection, we propose a scheme to evaluate the channel quality using these estimated parameters.

\section{B. Channel Quality Prediction}

In our work, we employ two metrics to evaluate the channel quality. The first one is the spectrum sensing accuracy of the secondary user on the primary channel, which has influence on the spectrum utilization efficiency of the secondary user; the second one is the expected duration of the idle state, which implies the channel availability to the secondary user.

First, we design an evaluation metric for the spectrum sensing accuracy. It can be seen from [29] that a higher detection probability $P_{d}$ always comes with a higher false alarm probability $P_{f}$. Therefore, $P_{d}\left(1-P_{f}\right)$ can be used to denote the spectrum sensing accuracy. In particular, IEEE 802.22 recommends that the false alarm probability $\left(P_{f}\right)$ and the miss detection probability $\left(1-P_{d}\right)$ should be lower than 0.1 [30]. Thus we assume $P_{f} \in[0.01,0.1]$ and $P_{d} \in[0.9,0.99]$. As a result, $P_{d}\left(1-P_{f}\right) \in[0.81,0.99]$.

In Section III-B, we point out that $b_{01}=P_{f}$ and $b_{11}=$ $P_{d}$. Thus $\hat{B}_{N}$ can be used to estimate the spectrum sensing accuracy. Let $\mathrm{M}_{A}$ be the evaluation metric for the spectrum sensing accuracy. Then

$$
\mathrm{M}_{A}=\widehat{b}_{11}\left(1-\widehat{b}_{01}\right)
$$

where $\widehat{b}_{11}$ and $\widehat{b}_{01}$ denote the estimated detection probability and false alarm probability, respectively.

Second, we define $\mathrm{M}_{E}=1 / \widehat{\lambda}_{0}$ to denote the estimated idle duration of the primary channel.
Finally, the channel quality $\mathrm{M}_{Q}$ can be estimated by combing the two metrics $\mathrm{M}_{A}$ and $\mathrm{M}_{E}$. Let

$$
\mathrm{M}_{Q}=\left(1+\log _{\epsilon} \mathrm{M}_{A}\right) \mathrm{M}_{E}
$$

where $\epsilon>1$ is a parameter indicating the preference of the SU when estimating the quality of the primary channel. It can be derived that

$$
\begin{gathered}
\frac{\partial \mathrm{M}_{Q}}{\partial \mathrm{M}_{A} \partial \epsilon}=-\frac{\mathrm{M}_{A}}{\mathrm{M}_{E} \epsilon}\left(\frac{1}{\ln \epsilon}\right)^{2}<0 \\
\frac{\partial \mathrm{M}_{Q}}{\partial \mathrm{M}_{E} \partial \epsilon}=-\ln \mathrm{M}_{A}\left(\frac{1}{\ln \epsilon}\right)^{2}>0
\end{gathered}
$$

Therefore, when predicting the channel quality $\mathrm{M}_{Q}$, SU could choose a bigger $\epsilon$ to place more importance on $\mathrm{M}_{E}$ or a smaller $\epsilon$ to give more preference to $\mathrm{M}_{A}$.

\section{Simulation}

In this section, we evaluate the performance of the proposed scheme for channel quality prediction through simulation study. The effect of the parameters, namely the estimated spectrum sensing accuracy $\mathrm{M}_{A}$, the estimated idle duration $\mathrm{M}_{E}$, and the preference parameter $\epsilon$, on the estimated channel quality $\mathrm{M}_{Q}$, are extensively investigated. In particular, the effectiveness of our design is studied through two example application scenarios. It should be noticed that our simulation

TABLE I

SimUlation SetTings

\begin{tabular}{|c|c|}
\hline Parameter & Value \\
\hline Length of the state/observation sequence $(T)$ & 100 \\
\hline False alarm probability $\left(P_{f}\right)$ & {$[0.01,0.1]$} \\
\hline Detection probability $\left(P_{d}\right)$ & {$[0.9,0.99]$} \\
\hline $\begin{array}{c}\text { Rate parameter of the exponential } \\
\text { distribution }\left(\lambda_{i}, i=0,1\right)\end{array}$ & {$[0.02,1]$} \\
\hline Preference parameter $(\epsilon)$ & {$[1.1,10.0]$} \\
\hline
\end{tabular}

is not conducted over a physical network model since our work does not rely on any physical layer setting. In a cognitive radio system, each SU has a detection probability $P_{d}$ and a false alarm probability $P_{f}$ on a primary channel. The only assumption adopted by our work is that the primary channel states (busy/idle) follow Exponential distributions, which has been validated by many previous research [24], [25]. Following our derivations, each SU can estimate the parameters through Bayesian inference and then predict the channel quality using the estimated parameters. In this simulation study, the state sequences and the corresponding observation sequences are generated from the parameters listed in TABLE I. Each SU performs Bayesian inference with Gibbs sampling according to the procedure described in Section $\mathrm{V}-\mathrm{A}$, and predicts the quality of the channels according to (34).

Note that we have tried different values of $\epsilon$ ranging from 1.1 to 10.0 but will report only the results when $\epsilon \epsilon$ $\{1.2,1.3,1.4,7.0\}$ to demonstrate the trend of the influence of $\epsilon$ on the performance of our design. 


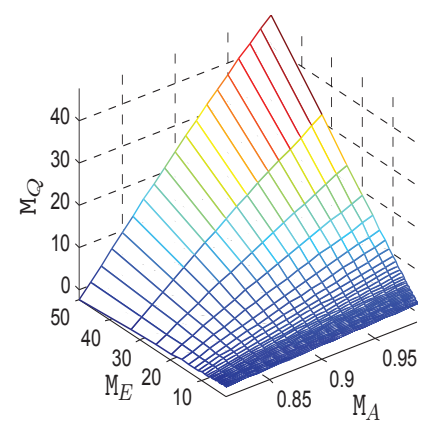

(a) $\epsilon=1.2$

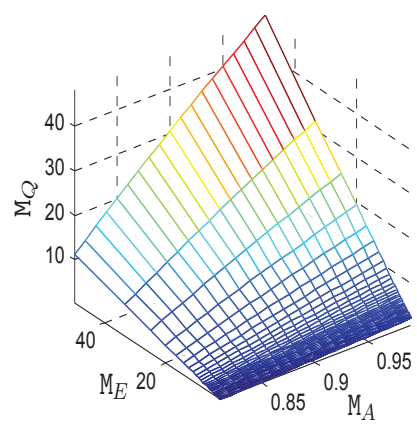

(b) $\epsilon=1.3$

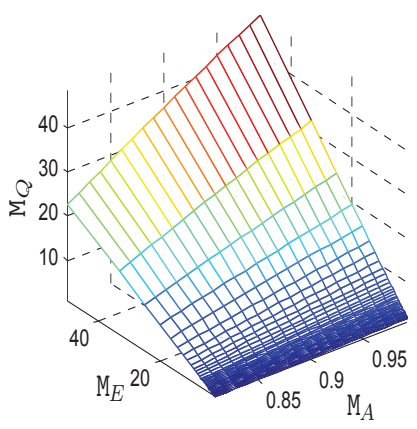

(c) $\epsilon=1.4$

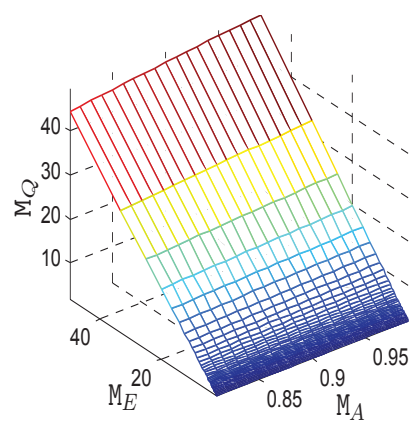

(d) $\epsilon=7$

Fig. 3. The effect of the estimated system parameters on the channel quality.

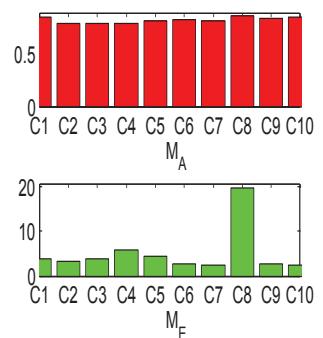

(a) Estimated $\mathrm{M}_{A}$ and $\mathrm{M}_{E}$

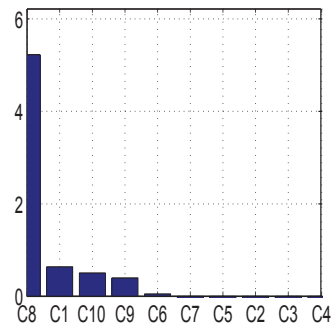

(b) $\epsilon=1.2$

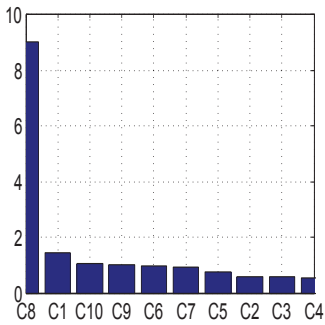

(c) $\epsilon=1.3$

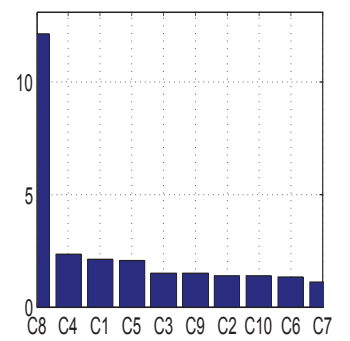

(d) $\epsilon=1.4$

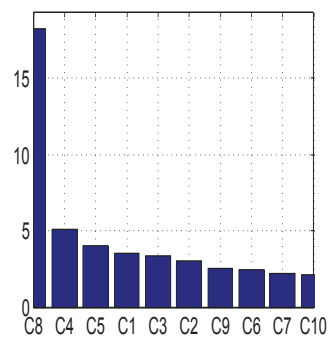

(e) $\epsilon=7$

Fig. 4. The channel ranking based on the channel quality prediction.

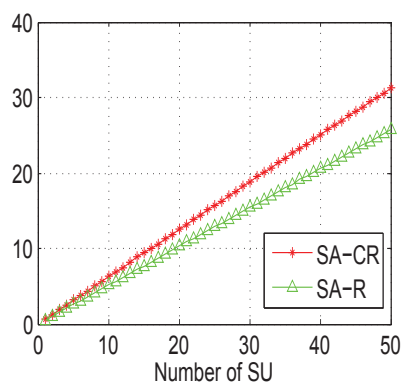

(a) Average explored transmission opportunities per time slot

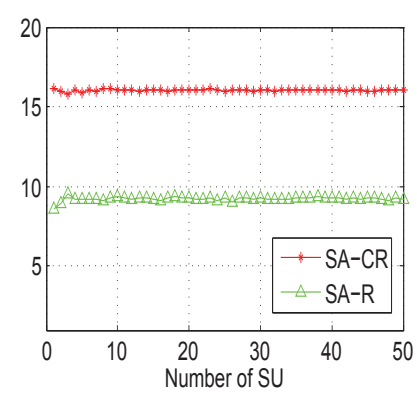

(b) Average idle state duration

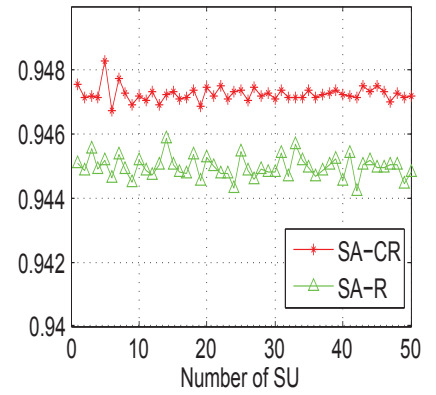

(c) Average detection probability

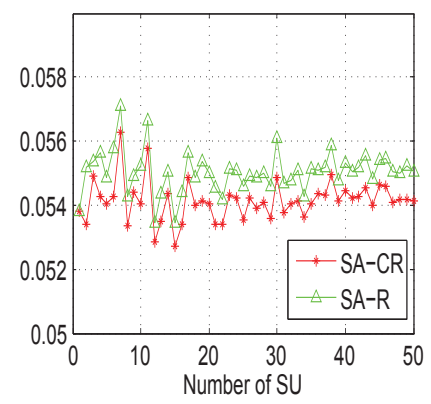

(d) Average false alarm probability

Fig. 5. The number of SUs $v s$. the explored transmission opportunities.

\section{A. The Effect of Estimated System Parameters}

In this subsection, we study the effect of the estimated system parameters on the channel quality. According to the previous analysis, it is reasonable to assume that the values of the estimated parameters fall into the following fixed ranges: $\widehat{b}_{11} \in[0.9,0.99], \widehat{b}_{01} \in[0.01,0.1]$, and $\widehat{\lambda}_{i} \in[0.02,1]$, where $i=0,1$. Therefore, $\mathrm{M}_{A} \in[0.81,0.99]$ and $\mathrm{M}_{E} \in[1,50]$.

Fig. 3 reports the effect of the estimated spectrum sensing accuracy $\mathrm{M}_{A}$ and the idle duration $\mathrm{M}_{E}$ on the channel quality. Obviously, $\mathrm{M}_{Q}$ is an increasing function of both $\mathrm{M}_{A}$ and $\mathrm{M}_{E}$. This result indicates that a channel exhibits higher quality if it can provide more transmission opportunities to the secondary user and/or the secondary user can detect these opportunities more accurately.

Figs. 3(a), 3(b), 3(c), 3(d) provide an intuitive explanation regarding the effect of the preference parameter $\epsilon$ on the channel quality. When $\epsilon$ is small, the SU places more importance on the spectrum sensing accuracy; therefore $M_{A}$ has great influence on $\mathrm{M}_{Q}$. A channel with a lower $\mathrm{M}_{A}$ must provide more available idle opportunities (a bigger $\mathrm{M}_{E}$ ) to gain a higher $\mathrm{M}_{Q}$. However, the influence of $\mathrm{M}_{A}$ is gradually weakened with the increase of $\epsilon$. When $\epsilon=7, \mathrm{M}_{A}$ has quite small influence on $\mathrm{M}_{Q}$. In this case, the channel quality is almost completely determined by its availability $\mathrm{M}_{E}$.

\section{B. Application Scenario 1: Channel Ranking When Multiple Primary Channels Exist}

In cognitive radio networks, it is important to rank the channels according to the predicted channel quality and form an ordered sequence for spectrum sensing. Thus in this subsection, we investigate the performance of channel ranking 
based on the proposed channel quality prediction mechanism when multiple primary channels exist. Since the idle duration of a primary channel, i.e., the length of the time interval the primary channel stays idle in the channel, is a critical parameter in evaluating the channel quality, we consider a network scenario with one SU and $N$ PUs, with each of which possessing one primary channel. The primary users are denoted by $P U_{1}, P U_{2}, \cdots, P U_{N}$ and the corresponding primary channels are denoted by $C_{1}, C_{2}, \cdots, C_{N}$. Our simulation is conducted on an example scenario with $N=10$, but our results can be readily generalized to the case when $N$ is any arbitrary positive integer. The parameters of the channels, and the detection probabilities and false alarm probabilities of the SU over the channels, are randomly selected and the settings are given in TABLE II. Note that the false alarm probabilities of the SU on different channels are the same since $P_{f}$ mainly depends on the threshold (sensitivity) of the energy detector.

TABLE II

The Parameters of the Ten Channels

\begin{tabular}{|c|c|c|c|c|c|}
\hline Channel & $C_{1}$ & $C_{2}$ & $C_{3}$ & $C_{4}$ & $C_{5}$ \\
\hline$\lambda_{0}$ & 0.26 & 0.29 & 0.26 & 0.17 & 0.22 \\
\hline$\lambda_{1}$ & 0.49 & 0.33 & 0.35 & 0.21 & 0.14 \\
\hline$P_{d}$ & 0.97 & 0.91 & 0.91 & 0.91 & 0.93 \\
\hline$P_{f}$ & 0.09 & 0.09 & 0.09 & 0.09 & 0.09 \\
\hline Channel & $C_{6}$ & $C_{7}$ & $C_{8}$ & $C_{9}$ & $C_{10}$ \\
\hline$\lambda_{0}$ & 0.36 & 0.41 & 0.05 & 0.36 & 0.43 \\
\hline$\lambda_{1}$ & 0.17 & 0.05 & 0.05 & 0.1 & 0.24 \\
\hline$P_{d}$ & 0.94 & 0.92 & 0.99 & 0.96 & 0.98 \\
\hline$P_{f}$ & 0.09 & 0.09 & 0.09 & 0.09 & 0.09 \\
\hline
\end{tabular}

Fig. 4(a) reports the estimated $\mathrm{M}_{A}$ and $\mathrm{M}_{E}$ of the primary channels. Taking both the spectrum sensing accuracy (indicated by $\mathrm{M}_{A}$ ) and the idle duration of the channel (indicated by $\mathrm{M}_{E}$ ) into consideration, SU estimates the quality of the channels and rank them in the non-increasing order of $\mathrm{M}_{Q}$. Fig. 4(b), 4(c), 4(d), 4(e) illustrate the results of channel ranking when $\epsilon=1.2,1.3,1.4$, and 7 , respectively. It can be seen that the ordered channel sequence changes with $\epsilon$. When $\epsilon$ is small, $\mathrm{M}_{A}$ plays an important role in determining the channel quality; thus channels with higher $\mathrm{M}_{A}$ values are ordered in the top of the sequence. However, when $\epsilon$ is big, the channel quality mainly depends on the availability $\mathrm{M}_{E}$; thus the channels with higher $\mathrm{M}_{E}$ values are placed at the top of the sequence.

\section{Application Scenario 2: Dynamic Spectrum Access Based on Channel Ranking When Multi-SU Multi-channel Exist}

In this subsection, we investigate the performance of dynamic spectrum access based on the channel ranking obtained from channel quality prediction. In channel ranking, each $\mathrm{SU}$ forms an ordered channel sequence, denoted as $\mathfrak{C}_{r}=$ $\left\{C_{r_{1}}, C_{r_{2}}, \cdots, C_{r_{N}}\right\}$, where $r_{i} \in\{1, \cdots, N\}$ and $N$ is the number of primary channels in the network. Without loss of generality, we set $N=100$ and $\epsilon=1.4$ in the following simulation study.

There are two types of spectrum access mechanisms: Spectrum Access based on Random channel selection (SA-
R) in which each SU randomly selects one channel to sense and access at each time slot; and Spectrum Access based on Channel Ranking (SA-CR) in which each SU maintains an ordered channel sequence and sequentially sense and access the channels based on the channel ranking. SA-R is a commonly adopted spectrum access scheme in cognitive radio networks [17], [18], [31]. In the following we compare the performance of SA-CR and SA-R from three different aspects.

First, we consider a simple scenario where all the secondary users keep on sensing the states of the channels to explore possible transmission opportunities. We say "a SU captures a transmission opportunity" if it correctly detects the idle state of a channel at the beginning of a time slot. When a transmission opportunity is explored, the SU starts its transmission (facilitated by multiple access control) until the end of the slot. Taking into account the fact that the available channels are usually more than the number of users for typical CRN applications [32], we vary the number of SUs from 1 to $\frac{N}{2}$ in our simulation.

It can be seen from Fig. 5(a) that the captured transmission opportunities in both SA-CR and SA-R increase with the number of SUs in the network. When there are few SUs, some channels can not be utilized. The possible transmission opportunities can be better excavated with more SUs. Moreover, Figs 5(b), 5(c) and 5(d) indicate that the channel quality of the explored transmission opportunities in SA-CR are better than that in SA-R, in terms of the expected idle duration, the detection probability, and the false alarm probability.

We also carry out a simulation study to investigate the performance of SA-CR and SA-R on the successful transmission rate of the SUs and the average time cost for finding available channels when the arrivals of the SUs follow a Poisson distribution. Fig. 6 reports the impact of the arrival rate on the performance of SA-CR and SA-R. It is obvious that taking SA-CR instead of SA-R can significantly improve the successful transmission rate and reduce the time cost for each SU to find an available channel.

\section{CONCLUSION}

In this paper, we study the problem of channel quality prediction in cognitive radio networks. The proposed approach adaptively infer the system parameters and then estimate the channel quality based on the inferred parameters. We design a channel quality metric $\mathrm{M}_{Q}$, which takes into account both the spectrum sensing accuracy and the expected channel idle duration time. Our future research involves further extensive empirical investigations and analytical studies on the proposed approach.

\section{ACKNOWLEDGMENT}

The authors would like to thank the support from the National Natural Science Foundation of China (Grant No. 61272503, 61272505 and 61172074), the Fundamental Research Funds for the Central Universities of China (2012YJS007), and the National Science Foundation of the US (CNS-1162057). 


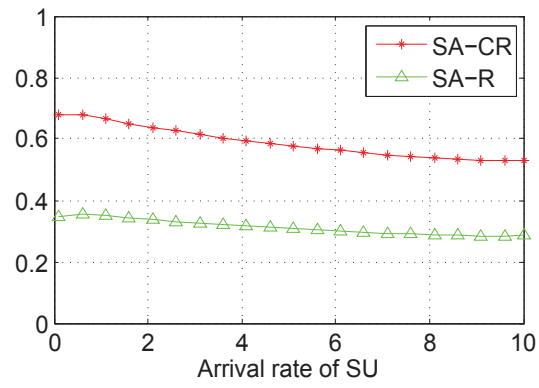

(a) Successful transmission rate

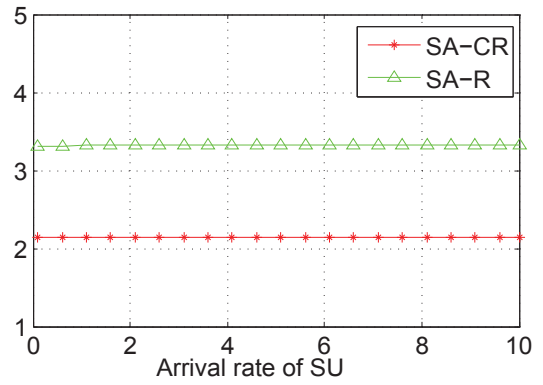

(b) Average time cost (in time slot) for finding available channel

Fig. 6. The impact of SUs' arrival rate.

\section{REFERENCES}

[1] M. Song, C. Xin, Y. Zhao, and X. Cheng, "Dynamic spectrum access: From cognitive radio to network radio," IEEE Wireless Communications, vol. 19, no. 1, pp. 23-29, February 2012.

[2] T. Jing, X. Chen, Y. Huo, and X. Cheng, "Achievable transmission capacity of cognitive mesh networks with different media access control," in IEEE INFOCOM, March 2012, pp. 1764-1772.

[3] S. Wang, J. Zhang, and L. Tong, "Delay analysis for cognitive radio networks with random access: A fluid queue view," in IEEE INFOCOM, March 2010, pp. 1-9.

[4] X. Wang, Z. Li, P. Xu, Y. Xu, X. Gao, and H.-H. Chen, "Spectrum sharing in cognitive radio networks-an auction-based approach," IEEE Transactions on Systems, Man, and Cybernetics, Part B: Cybernetics, vol. 40, no. 3, pp. 587-596, June 2010.

[5] L. Gao, X. Wang, Y. Xu, and Q. Zhang, "Spectrum trading in cognitive radio networks: A contract-theoretic modeling approach," IEEE Journal on Selected Areas in Communications (JSAC), vol. 29, no. 4, pp. 843855, April 2011.

[6] W. Li, X. Cheng, T. Jing, Y. Cui, K. Xing, and W. Wang, "Spectrum assignment and sharing for delay minimization in multi-hop multi-flow crns," IEEE Journal on Selected Areas in Communications (JSAC), Special Issue on Cognitive Radio, March 2013.

[7] W. Li, X. Cheng, T. Jing, and X. Xing, "Cooperative multi-hop relaying via network formation games in cognitive radio networks," in IEEE INFOCOM, 2013.

[8] H. Li, X. Cheng, K. Li, X. Xing, and T. Jing, "Utility-based cooperative spectrum sensing scheduling in cognitive radio networks," in IEEE INFOCOM Mini-Conference, 2013.

[9] T. Jing, S. Zhu, H. Li, X. Cheng, and Y. Huo, "Cooperative relay selection in cognitive radio networks," in IEEE INFOCOM Mini-Conference, 2013.

[10] Z. Chen, N. Guo, Z. Hu, and R. Qiu, "Experimental validation of channel state prediction considering delays in practical cognitive radio," IEEE Transactions on Vehicular Technology, vol. 60, no. 4, pp. $1314-1325$, May 2011.

[11] V. K. Tumuluru, P. Wang, and D. Niyato, "Channel status prediction for cognitive radio networks," Wireless Communications and Mobile Computing, vol. 12, no. 10, pp. 862-874, 2012.

[12] Y. Li, N. Seshadri, and S. Ariyavisitakul, "Channel estimation for ofdm systems with transmitter diversity in mobile wireless channels," IEEE Journal on Selected Areas in Communications (JSAC), vol. 17, no. 3, pp. $461-471$, March 1999.

[13] K. Balachandran, S. Kadaba, and S. Nanda, "Channel quality estimation and rate adaptation for cellular mobile radio," IEEE Journal on Selected Areas in Communications (JSAC), vol. 17, no. 7, pp. $1244-1256$, July 1999.

[14] G. Oien, H. Holm, and K. Hole, "Impact of channel prediction on adaptive coded modulation performance in rayleigh fading," IEEE Transactions on Vehicular Technology, vol. 53, no. 3, pp. 758 - 769, May 2004.

[15] N. Nie and C. Comaniciu, "Adaptive channel allocation spectrum etiquette for cognitive radio networks," in IEEE DySPAN, November 2005, pp. $269-278$.
[16] D. Niyato and E. Hossain, "Competitive pricing for spectrum sharing in cognitive radio networks: Dynamic game, inefficiency of nash equilibrium, and collusion," IEEE Journal on Selected Areas in Communications (JSAC), vol. 26, no. 1, pp. $192-202$, January 2008.

[17] D. Zheng, W. Ge, and J. Zhang, "Distributed opportunistic scheduling for ad hoc networks with random access: An optimal stopping approach," IEEE Transactions on Information Theory, vol. 55, no. 1, pp. $205-222$, January 2009.

[18] P. Chandrashekhar Thejaswi, J. Zhang, M.-O. Pun, and H. Poor, "Distributed opportunistic scheduling with two-level channel probing," in IEEE INFOCOM, April 2009, pp. 1683 -1691.

[19] A. Fehske, J. Gaeddert, and J. Reed, "A new approach to signal classification using spectral correlation and neural networks," in IEEE DySPAN, November 2005, pp. $144-150$.

[20] F. Digham, M.-S. Alouini, and M. Simon, "On the energy detection of unknown signals over fading channels," in IEEE ICC, vol. 5, May 2003, pp. 3575-3579.

[21] D. Cabric, A. Tkachenko, and R. W. Brodersen, "Experimental study of spectrum sensing based on energy detection and network cooperation," in The 2nd Annual International Wireless Internet Conference (WICON). TAPAS Workshop, August 2006.

[22] C. Ghosh, C. Cordeiro, D. Agrawal, and M. Rao, "Markov chain existence and hidden markov models in spectrum sensing," in IEEE PerCom, March 2009, pp. 1 -6.

[23] P. Djuric and J.-H. Chun, "An mcmc sampling approach to estimation of nonstationary hidden markov models," IEEE Transactions on Signal Processing, vol. 50, no. 5, pp. 1113 -1123, May 2002.

[24] M. Wellens, J. Riihijarvi, and P. Mahonen, "Evaluation of adaptive mac-layer sensing in realistic spectrum occupancy scenarios," in IEEE DySPAN, April 2010, pp. $1-12$.

[25] A. Min, K.-H. Kim, J. Singh, and K. Shin, "Opportunistic spectrum access for mobile cognitive radios," in IEEE INFOCOM, April 2011, pp. $2993-3001$.

[26] S. Mao, J. Wang, and X. Pu, Advanced Mathematical Statistics. Higher Education Press, 2006.

[27] V. Nekoukhou, M. H. Alamatsaz, and H. Bidram, "A discrete analog of the generalized exponential distribution," Communications in Statistics - Theory and Methods, vol. 41, no. 11, pp. 2000-2013, 2012.

[28] J. Yan, "Bayesian survival analysis," Journal of the American Statistical Association, vol. 99, no. 468, pp. 1202-1203, 2004.

[29] W. Saad, Z. Han, M. Debbah, A. Hjorungnes, and T. Basar, "Coalitional games for distributed collaborative spectrum sensing in cognitive radio networks," in IEEE INFOCOM, April 2009, pp. 2114-2122.

[30] C. Stevenson, G. Chouinard, Z. Lei, W. Hu, S. Shellhammer, and W. Caldwell, "Ieee 802.22: The first cognitive radio wireless regional area network standard," IEEE Communications Magazine, vol. 47, no. 1, pp. $130-138$, January 2009.

[31] Y. Zhang, Q. Li, G. Yu, and B. Wang, "Etch: Efficient channel hopping for communication rendezvous in dynamic spectrum access networks," in IEEE INFOCOM, April 2011, pp. $2471-2479$.

[32] M. Mishra and A. Sahai, "How much white space is there?" EECS Department, University of California, Berkeley, Tech. Rep. UCB/EECS-2009-3, Jan 2009. [Online]. Available: http://www.eecs.berkeley.edu/Pubs/TechRpts/2009/EECS-2009-3.html 